\title{
NUCLEAR ENERGY RESEARCH INITIATIVE PROGRAM (NERI) QUARTERLY PROGRESS REPORT - New Design Equations for Swelling and Irradiation Creep in Generation IV Reactors
}

\author{
M.P. Surh, W.G. Wolfer
}

U.S. Department of Energy

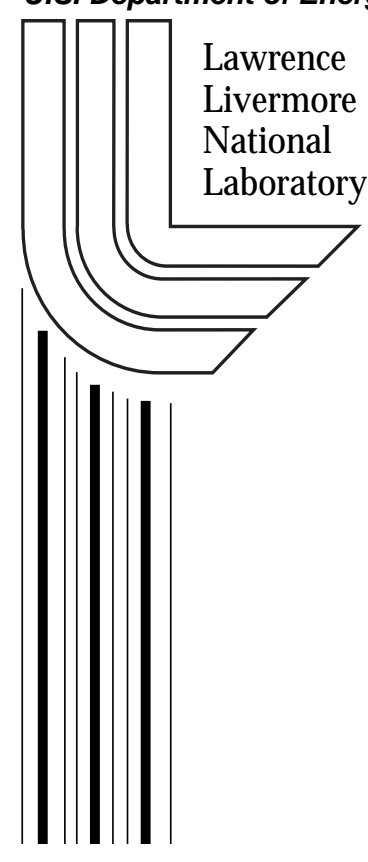

July 25, 2003 


\section{DISCLAIMER}

This document was prepared as an account of work sponsored by an agency of the United States Government. Neither the United States Government nor the University of California nor any of their employees, makes any warranty, express or implied, or assumes any legal liability or responsibility for the accuracy, completeness, or usefulness of any information, apparatus, product, or process disclosed, or represents that its use would not infringe privately owned rights. Reference herein to any specific commercial product, process, or service by trade name, trademark, manufacturer, or otherwise, does not necessarily constitute or imply its endorsement, recommendation, or favoring by the United States Government or the University of California. The views and opinions of authors expressed herein do not necessarily state or reflect those of the United States Government or the University of California, and shall not be used for advertising or product endorsement purposes.

Work performed under the auspices of the U. S. Department of Energy by the University of California Lawrence Livermore National Laboratory under Contract W-7405-Eng-48.

This report has been reproduced

directly from the best available copy.

Available to DOE and DOE contractors from the

Office of Scientific and Technical Information

P.O. Box 62, Oak Ridge, TN 37831

Prices available from (423) 576-8401

$$
\text { http://apollo.osti.gov/bridge/ }
$$

Available to the public from the

National Technical Information Service

U.S. Department of Commerce 5285 Port Royal Rd., Springfield, VA 22161 http://www.ntis.gov/

OR

Lawrence Livermore National Laboratory Technical Information Department's Digital Library http://www.llnl.gov/tid/Library.html 


\title{
NUCLEAR ENERGY RESEARCH INITIATIVE PROGRAM (NERI) QUARTERLY PROGRESS REPORT
}

\author{
New Design Equations for Swelling and Irradiation Creep \\ in Generation IV Reactors
}

Project Number: 01-137

Quarter (5) Report

April-June 2003

\author{
Compiled by: \\ Lead Organization: Lawrence Livermore National Laboratory \\ Michael P. Surh \\ P.I. \\ W.G. Wolfer
}

Collaborating Organizations:

Pacific Northwest National Laboratory, Richland, WA

University of California, Berkeley, CA

Submitted on:

July 25, 2003 


\section{NERI Quarterly Progress Report \\ New Design Equations for Swelling and Irradiation Creep \\ in Generation IV Reactors \\ Project Number: 01-137 \\ April-June 2003}

\section{A. Task A: Void Nucleation (LLNL)}

1.Task Status and Significant Results:

a) We have submitted our paper on computational methods for void nucleation and growth, "Master equation and Fokker-Planck methods for void nucleation and growth in irradiation swelling", by Michael P. Surh, J. B. Sturgeon, and W. G. Wolfer, to The Journal of Nuclear Materials.

b) We have completed two additional papers on void nucleation and growth with and without dislocation co-evolution. We find that the co-evolution of dislocation content enhances the quasi-steady swelling behavior at late times. The predicted steady-swelling rate lies near $1 \% /$ dpa because the underlying defect production rate $(0.1$ Frenkel pair per dpa) and stress-induced bias factors reproduce the experimentally observed rate of vacancy/interstitial segregation for the high ratio of sink strengths. The swelling is quasisteady because the ratio of void defect sinks to dislocation sink strength stays in a narrow post-incubation range (near 2:1 to 4:1) for the length of the simulation (up to $100 \mathrm{dpa}$ ) and for a wide range of temperature. Two factors help preserve this ratio. First, for most temperatures, fewer stable voids nucleate when the initial dislocation content is low. When few and large voids increase their volume during irradiation swelling, their aggregate sink strength remains more nearly constant than when many and small voids grow by the same net volume. Thus, solution-annealed initial conditions better preserve the ratio of void to dislocation sink strengths over subsequent volume changes. Second, the solution-annealed dislocation density increases to a terminal value (typically by a factor of 10) after the stable voids have nucleated. This makes the ratio of sink strengths even less sensitive to the chronic void growth and thereby prolongs the quasi-steady swelling behavior.

c) We have completed extensive calculations on incubation times versus temperature, dose-rate, starting dislocation density, and model dislocation bias factors. The results are being analyzed to understand the incubation peak seen at intermediate temperatures in some models.

2. Issues/Concerns:

a) None.

b) None.

c) The discrepancies between simulations with a constant dislocation bias factor and those with a density-dependent dislocation bias factor emphasize the need for an 
improved, detailed treatment of dislocation content in terms of loops, isolated network dislocations, and dislocation cell walls, with their own bias factors and sink strengths. Realistic simulations require that several subtasks (that fall under the category of Tasks B and D) be completed.

\section{B. Task B: Dislocation Loop Formation and Dislocation Network Evolution (LLNL)}

1. Task Status and Significant Results:

a) We have finished code development to treat the fundamental problem of defectcluster coalescence. We have separately implemented Gillespie's Markov Monte Carlo procedure for aggregation of discrete clusters (work performed over this and the preceding quarter). We also incorporated several algorithmic improvements to a standalone version of Gillespie's method in this quarter. The computational demands of our new implementation scale better versus the number of Monte Carlo particles, making our calculations substantially faster in practical applications.

We have extended the Gillespie algorithm to treat so-called macroparticles, a concept that we only recently encountered. This is a numerical technique widely used in plasma physics applications to model non-equilibrium distribution functions. (Coincidentally, our void nucleation and growth simulations already use this approach, since it is a natural solution to modeling the dynamics of the distribution function.) The macroparticle approximation allows our Monte Carlo method to be used in conjunction with a standard rate-theory calculation for small clusters. It also makes the method applicable to systems with widely varying concentrations of the various species (cluster sizes), unlike the original Gillespie method.

We have joined this Markov Monte Carlo method with our rate-theory implementation (that uses VODE) developed during the previous quarter. We now possess three methods for treating cluster growth with coalescence: a pure-rate theory method with VODE, a pure Monte Carlo method, and a hybrid that uses rate-theory for small clusters and Monte Carlo for large. The three methods produce substantially the same results, providing a useful consistency check on the different calculations. Very preliminary tests of the hybrid method for irreversible cluster growth are encouraging and indicate that our simulations can continue to be performed on single processor platforms. The overarching computational treatment of microstructural evolution under irradiation is developing satisfactorily.

b) We have previously found that the loop-loop capture distance depends on torques originating from the stress field, which can be changed by external stresses. Ultimately, this stress-dependent loop annihilation and aggregation must include the network dislocations, to complete a process that can drive irradiation creep. As the next step, we are developing a model of loop-network dislocation evolution. The network dislocation possesses longer-range stress fields, so a stronger interaction with loops is expected.

2. Issues/Concerns:

a) The coalescence method will be first applied to helium bubble evolution (Task D) in the next quarter. Acceptable models for bubble impingement cross-sections and sizedependent bubble mobilities are already available. The resulting processes of helium bubble growth will be evaluated by comparing simulations to experiment. Bubble 
formation is itself an important damage issue in reactor materials. TEM measurements from HFIR and thermal reactor steels as well as plutonium samples suggest that helium bubbles reach a maximum radius of around $2 \mathrm{~nm}$; relatively few bubbles seem to grow larger. Instead, a high density of new, small bubbles develops with further helium generation. These dispersed pinning points raise the yield stress and increase irradiation embrittlement.

This apparent experimental observation of a maximum bubble size is not consistent with the simplest size-dependent cluster diffusion model. This possible discrepancy poses an interesting puzzle. Accurate evaluations of the simplest coalescence evolution models will clarify any potential differences with experiment. The explanation of these observations may require additional modeling or experimental study.

Our new hybrid method for cluster aggregation appears to be practical for simulating irreversible cluster growth. It should still apply when cluster evaporation simultaneously occurs (as with vacancy clusters). However, the shrinkage of the Monte Carlo clusters must be treated more carefully than in our existing nucleation and growth code. The new rate-theory treatment will be applied to a smaller range of cluster sizes because VODE is a slower than our old, Euler method. This means that a higher proportion of the Monte Carlo macroparticles that are created will shrink and disappear in the new calculations. A likeliest solution to this shrinkage problem has been identified, but it must be tested to ensure computational feasibility.

b) The modeling of coalescence during dislocation loop evolution still awaits the appropriate bias factors and sink strengths plus realistic rate coefficients for the coalescence of loops. We may undertake preliminary simulations with idealized reaction rates in order to optimize the program while the full reaction model is being developed.

\section{Task C: Dislocation-Void Interaction (UC-Berkeley and LLNL) \\ 1. Task Status:}

We have developed a higher order level set method for simulating the dynamics of dislocations moving in response to the Laplacian if curvature. This more accurate method results in more stable motion of the dislocation, but is still too inefficient to be useful for larger scale simulations. We are exploring methods to improve the efficiency.

2. Issues/Concerns:

None.

D. Task D: Model Integration (LLNL)

1. Task Status:

a) We are examining the possibility of defect cluster dissolution by cascade engulfment by surveying MD calculations of cascade/cavity overlap. This is potentially a relevant mechanism for broadening the size distribution of voids or limiting the size of helium bubbles (see Issues/Concerns in Task B for the empirically observed maximum helium bubble sizes). For example, cascade overlap could destroy or shrink the older bubbles in the material. Alternatively, it could destroy only the smaller clusters, prolonging the time to nucleate stable voids. If microscopic modeling confirms the importance of cluster dissolution or fission by irradiation bombardment, then it is already 
feasible to include the processes in the VODE/Monte Carlo implementation of cluster evolution.

b) We are examining the effect of stress pulses on the mobility of prismatic dislocation loops. Such disturbances could result from the thermal pulse of an irradiation cascade. Stress and temperature excursions could also reduce the effective barriers to loop motion by activating motion over the barriers.

2. Issues/Concerns:

None.

E. Task E: Validation (PNNL and LLNL)

1. Task Status:

a) We have submitted the paper, "A Critical Test of the Classical Rate Theory for Void Swelling", by T. Okita and W.G. Wolfer, to The Journal of Nuclear Materials.

b) We have expanded our survey of dose-rate effects to include other parameters. The paired FFTF experiments on ferritic and austenitic alloys that we have analyzed merits this effort. In these experiments, the bcc materials showed no dose rate effect, while the fcc samples in the same packets did show a dose-rate effect. Bcc bias factors are believed to be smaller, based on microscopic theory and on the smaller steady swelling rate $(0.2 \% / \mathrm{dpa})$. In general, void nucleation is harder in the bcc case. As a result, the effects of helium generation are more important. Based on the exposures of the samples, it is our understanding that the flux effects in bec are simply overwhelmed by the opposite influence of helium generation.

c) An important new effort is to experimentally test swelling/creep interaction in a real, constrained geometry. Non-uniform irradiation and temperature profiles will lead to uneven swelling and internal stresses. This will be partially relieved by irradiation creep, so that the swelling and creep will interact. Such interplay will be relevant to many thick structural components. To date, finite element modeling with existing swelling/creep constitutive relations has never been tested against a real experiment in thick samples.

The ideal experiment will employ thick samples of stainless steel, preferably 304, with gradients in temperature and irradiation flux. Experimental analysis will begin with nondestructive profilometry of the blocks and follow with sectioning and TEM sampling. This will provide a spatially-resolved representation of swelling and creep. A nearperfect realization of this has been found in an experiment from the EBR-II reflector involving three blocks of annealed type-304 steel, some 9.5 inches in length. These thick blocks were encased in $1 \mathrm{~mm}$ thin, hexagonal cans, and both were irradiated with flux and temperature gradients along their length and across the thick blocks. The surrounding cans underwent essentially stress-free swelling, while the blocks experienced constraint effects due to the nonuniform swelling and creep. Thus, the positiondependent swelling in the cans calibrates the position-dependence of the constrained swelling and creep. We have acquired these samples and are undertaking nondestructive measurements in preparation for detailed TEM sampling. The spatially-resolved data will be invaluable for testing future constitutive models for irradiation swelling and creep.

2. Issues/Concerns:

None. 
F. Task F: Development of Constitutive Equations (LLNL and PNNL)

1. Task Status:

a) We have submitted the paper, "Image Stresses of Dislocation Loops, Lenticular Inclusions, and Their Effects", by W.G. Wolfer, to Philosophical Magazine Letters.

This paper addresses effect of dislocation loops on dimensional changes in a finite body. There are experimental signs that the loop population undergoes a partial alignment under an applied stress. This loop alignment tends to feed on itself. It is necessary to correctly include the effect of loop-stress on the overall volume to simulate the effect on irradiation-enhanced creep.

b) We have begun assembling a paper discussing our three-sink model treatment of irradiation swelling.

2. Issues/Concerns:

None. 
$\underline{\text { Status Summary of NERI Tasks - Phase 1-3: }}$

\section{Phase1:}

\begin{tabular}{|l|l|l|}
\hline Milestone/Task Description & Planned Completion Date & Actual Completion Date \\
\hline Void nucleation with disloc. & Sept. 2002 & June 2002 \\
\hline Disl. Loop formation & Sept. 2002 & Sept. 2002 \\
\hline
\end{tabular}

\section{Phase2:}

\begin{tabular}{|l|l|l|}
\hline Milestone/Task Description & Planned Completion Date & Actual Completion Date \\
\hline $\begin{array}{l}\text { Preformed defect-clusters \& } \\
\text { Void Formation }\end{array}$ & August 2003 & postponed to Phase 3. \\
\hline $\begin{array}{l}\text { Incubation for SS304 \& } \\
\text { Pure Alloys }\end{array}$ & May 2003 & Mar. 2003 \\
\hline $\begin{array}{l}\text { Coupled Loop and Network } \\
\text { Evolution }\end{array}$ & June 2003 & postponed to Phase 3 \\
\hline $\begin{array}{l}\text { Cluster coalescence } \\
\text { modeling (He bubbles) }\end{array}$ & Sept. 2003 & \\
\hline $\begin{array}{l}\text { Tabulation of MD cascade } \\
\text { debris cluster distributions }\end{array}$ & Sept. 2003 & \\
\hline $\begin{array}{l}\text { Functional Forms for } \\
\text { Constitutive Equations }\end{array}$ & Sept. 2003 & \\
\hline
\end{tabular}

\section{Phase 3:}

\begin{tabular}{|l|l|l|}
\hline Milestone/Task Description & Planned Completion Date & Actual Completion Date \\
\hline $\begin{array}{l}\text { Joint bubble-void } \\
\text { simulations }\end{array}$ & Dec. 2003 & \\
\hline $\begin{array}{l}\text { Void growth with } \\
\text { preformed loop defects }\end{array}$ & Dec. 2003 & \\
\hline $\begin{array}{l}\text { Void growth plus loop } \\
\text { growth simulations }\end{array}$ & Feb. 2004 & \\
\hline $\begin{array}{l}\text { Joint bubble/void } \\
\text { loop/dislocation simulations }\end{array}$ & May. 2004 & \\
\hline
\end{tabular}

